\title{
Radiation damages during synchrotron X-ray micro-analyses of Prussian blue and zinc white historic paintings: detection, mitigation and integration
}

\author{
Claire Gervais $^{1} \cdot$ Mathieu Thoury $^{2} \cdot$ Solenn Réguer $^{3} \cdot$ Pierre Gueriau $^{3} \cdot$ \\ Jennifer Mass ${ }^{4}$
}

Received: 24 April 2015/ Accepted: 28 August 2015/Published online: 9 September 2015

(C) Springer-Verlag Berlin Heidelberg 2015

\begin{abstract}
High-flux synchrotron techniques allow microspectroscopic analyses of artworks that were not feasible even a few years ago, allowing for a more detailed characterization of their constituent materials and a better understanding of their chemistry. However, interaction between high-flux photons and matter at the sub-microscale can generate damages which are not visually detectable. We show here different methodologies allowing to evidence the damages induced by microscopic X-ray absorption near-edge structure spectroscopy analysis ( $\mu$ XANES) at the Fe and Zn K-edges of a painting dating from the turn of the twentieth century containing Prussian blue and zinc white. No significant degradation of the pigments was noticed, in agreement with the excellent condition of the painting. However, synchrotron radiation damages occurred at several levels, from chemical changes of the binder, modification of crystal defects in zinc oxide, to Prussian blue photoreduction. They could be identified by using both the $\mu$ XANES signal during analysis and with photoluminescence imaging in the deep ultraviolet and visible ranges after analysis. We show that recording
\end{abstract}

Claire Gervais

claire.gervais@hkb.bfh.ch

1 Bern University of the Arts, Fellerstrasse 11, 3027 Bern, Switzerland

2 IPANEMA, USR 3461 CNRS/MCC, Synchrotron SOLEIL, BP48 Saint-Aubin, 91192 Gif-sur-Yvette, France

3 Synchrotron SOLEIL, BP48 Saint-Aubin, 91192 Gif-sur-Yvette, France

4 Conservation Department, Winterthur Museum and Country Estate, Winterthur, DE 19735, USA accurately damaged areas is a key step to prevent misinterpretation of results during future re-examination of the sample. We conclude by proposing good practices that could help in integrating radiation damage avoidance into the analytical pathway.

\section{Introduction}

Microspectroscopic and microimaging approaches allow one to tackle the compositional and structural heterogeneity intrinsic to painting materials down to the submicroscale and are thus becoming more and more used for their investigation, whether it aims at characterizing pigments and binders used by the artist, highlighting possible degradations or understanding the physico-chemistry behind those degradation mechanisms [1-3]. Synchrotron techniques are particularly useful because the high brilliance of synchrotron light, access to small spot size and spatial coherence, allows one to collect data with a sufficient signal/noise ratio even for very small concentrations of materials, typically the only type of sample available from cultural heritage objects [4]. However, high photon fluxes may lead to radiation damage and several recent examples have pinpointed this problem [5-7].

Here, we present a typical case where radiation damage occurs for a sample exposed at typical photon dose under a microfocused synchrotron X-ray photons beam. A paint cross section from a turn of the twentieth century French early modernist painting was analysed by microscopic X-ray absorption near-edge structure spectroscopy ( $\mu$ XANES). Although the painting is in excellent condition, we were interested in analysing Prussian blue and zinc white pigments and possible alterations at the micro-scale resulting from their mixture. Indeed, Prussian blue has 
been shown in previous studies to readily fade when associated with white pigments such as zinc white, via a photocatalytic reaction still not well understood [8, 9]. $\mu \mathrm{XANES}$ at the $\mathrm{Zn}$ and Fe K-edges led, however, to different types of radiation-induced modifications observed during and after the sessions. We discuss the impact of these radiation damages on the information obtained, how they might lead to analytical misinterpretations, or to the contrary be integrated in our understanding of the material and its possible alterations. We finally conclude on experimental strategies to better detect, mitigate and integrate radiation damage and prevent the conflation of beam damage phenomena and the alteration resulting from the pigments' use and exhibition history.

\section{Methodology}

\subsection{Sample and preparation}

The sample was removed from the painting, embedded in Extec polyester resin, and a cross section was prepared and polished using MicroMesh $\mathrm{SiC}$ grinding papers (1500-12,000 grit). For electron microscopy, the sample was coated with conducting carbon paint (SPI supplies). Mock-up samples were prepared by the artist Valerio Cugia, with Prussian blue and zinc white directly extracted from the tubes (Talens-Rembrandt). Three mock-ups were prepared: pure Prussian blue (PB), Prussian blue diluted in zinc white with approximate volume ratio $1: 1(\mathbf{P B}+\mathbf{Z n})$ and 1:10 $(\mathbf{P b}++\mathbf{Z n})$.

\subsection{Microscopy}

Ultraviolet light microscopy was carried out using a Nikon Eclipse LV100 microscope at 20X Planfluor (N.A. 0.45). The excitation used was generated by using a mercury source (Nikon Intensilight C-HGFI) and fluorescence cube composed of a 450 to $490 \mathrm{~nm}$ excitation filter and $500 \mathrm{~nm}$ longpass filter for the detection. SEM-EDS data were collected using a Zeiss Evo MA electron microscope in variable pressure mode, with a lanthanum hexaboride filament and a Bruker Quantax 200 XFlash EDS detector. An accelerating voltage of $20 \mathrm{kV}$ was used, a beam current of $1.8 \mathrm{nA}$ and a working distance of $8.5 \mathrm{~mm}$.

\subsection{Raman spectroscopy}

Raman analysis was performed with a Raman spectrometer equipped with a 100x standing objective and a $780 \mathrm{~nm}$ diode laser. Laser power on the sample was limited to $200 \mu \mathrm{W}$ to avoid radiation damage. Exposure time was set to $2 \mathrm{~s}$ and 50 successive acquisitions were collected for each final spectrum.

\subsection{X-ray absorption spectroscopy}

$\mu \mathrm{XANES}$ measurements at the $\mathrm{Fe}$ and $\mathrm{Zn} \mathrm{K}$-edges were taken in fluorescence mode at the DiffAbs beamline at the SOLEIL synchrotron. The X-ray beam was collimated by two bendable mirrors, monochromatized using a Si(111) double-crystal monochromator (energy resolution of $0.7 \mathrm{eV}$ ) and focused using Kirkpatrick-Baez mirrors down to a spot size of $6 \times 6$ and $9 \times 6 \mu \mathrm{m}^{2}(\mathrm{H} \times \mathrm{V}$, full width at half maximum), respectively, at Fe and Zn K-edges. Photon flux at these energies was estimated at $1 \times 10^{10}$ photons/s in the focused spot. The fluorescence signal was monitored using a silicon drift detector (Vortex ME4) placed at $90^{\circ}$ to the beam in the horizontal plane. $\mu \mathrm{XANES}$ spectra at the $\mathrm{Fe}$ K-edge were collected in the 7090-7300 eV range with $0.5 \mathrm{eV}$ step size in the XANES region (7118.5-7145 eV). $\mu$ XANES spectra at the $\mathrm{Zn}$ K-edge were collected in the 9630-9800 eV range with $0.5 \mathrm{eV}$ step size in the XANES region (9661.5-9700 eV). The counting time was $1 \mathrm{~s}$ per energy step. Energy calibration was performed by using iron and zinc metallic foils, setting, respectively, the first inflection points of the edge at $7112 \mathrm{eV}$ and $9659 \mathrm{eV}$. XANES spectra were processed with the Athena software (Iffefit 1.2.9) [10]. The pre-edge range background was removed by subtracting a Victoreen function, and the absorption background was removed using a cubic spline with a normalization range between 30 and $260 \mathrm{eV}$ after the edge. The absorption edge was defined as the maximal of the first derivative.

\subsection{Synchrotron Deep-UV photoluminescence imaging}

The white beam of the DISCO beamline at the SOLEIL synchrotron was monochromatized (iHR320 monochromator, Jobin-Yvon Horiba, France) before insertion in a modified Zeiss Axio Observer Z1 microscope (Carl Zeiss, Germany). Images were collected at excitation wavelengths of $275 \mathrm{~nm}$ using a 300-nm reflecting dichroic beamsplitters (Omega Optical, USA) to reflect the incident monochromatic light upward through a Zeiss Ultrafluar 40X objective (Numerical aperture 0.6). Photoluminescence was collected through emission bandpass filters (Semrock, USA) positioned in front of a Princeton PIXIS camera (1024B/BUV, $1024 \times 1024$ pixels). The Diffraction PSF 3D plugin and Iterative Deconvolve 3D plugin from imageJ were used to generate theoretical PSFs (point spread function) and for iterative deblurring of the collected images, respectively. 


\section{Results}

The paint sample featured two overall compositions: a zinc white layer with lead white and barium sulphate inclusions, and a lead white paint with barium sulphate inclusions. A variety of high tinting strength pigments were added, including chrome yellow, Prussian blue and emerald green. A total of 21 spots were analysed at the Zn K-edge and 24 spots at the Fe K-edge in two successive beamtime sessions. The spots were chosen within layers where the intimate mixture between Prussian blue and zinc white was likely to occur (Fig. 1).

\subsection{Analysis of Prussian blue at Fe K-edge}

To evaluate the sensitivity of Prussian blue to radiation damage, two spectra were successively recorded on a spot rich in Prussian blue (Fig. 2). In the second scan, a significant shift of the absorption edge towards lower energies of $\approx 3 \mathrm{eV}$ is noticed, together with a slight increase in the second pre-peak and modifications of the first oscillation after the edge (stars). These spectral variations are in agreement with an X-ray-induced photoreduction in the pigment also observed in $\mu$ XANES studies of Prussian blue fibre systems $[11,12]$.

In previous studies, we showed that $\mathrm{X}$-ray radiation damage of Prussian blue could actually be positively exploited because the photoreduction induced by X-rays is similar to that induced by visible light, so that its sensitivity to X-rays may give some clues about its photosensitivity under visible light. Owing to this previous experience, we analysed further Prussian blue in different parts of the sample and in particular in the bulk and at the surface of Prussian blue aggregates, and in the vicinity of white pigments. We, however, reduced the number of acquired scans to 1 instead of 2 and decreased the number of energy steps to minimize exposure time and hence beam damage.
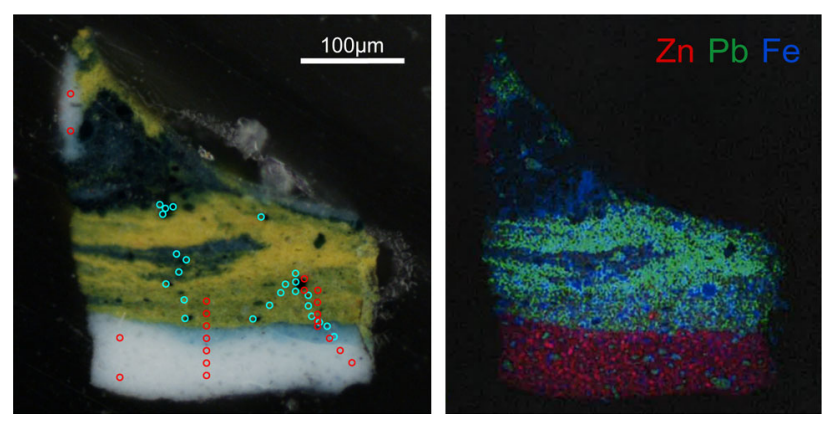

Fig. 1 Left: Overview of $\mu$ XANES measurements realized at the $\mathrm{Zn}$ K-edge (red) and Fe K-edge (cyan) on the paint sample. Right: SEMEDX image showing the distribution of zinc, iron and lead within the sample

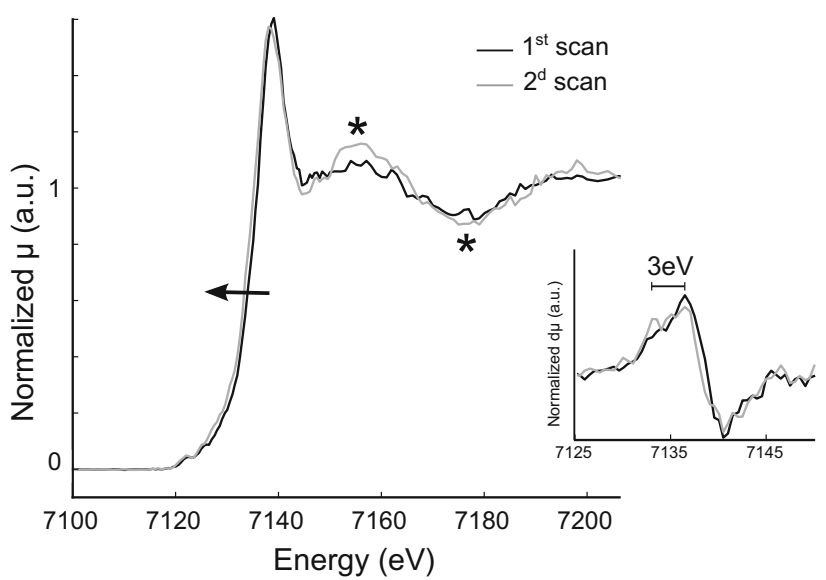

Fig. 2 X-ray radiation damage of Prussian blue observed during $\mu \mathrm{XANES}$ session at the Fe K-edge (denoted by arrow and stars). Inset: derivatives of the XANES spectra, showing a shift of the absorption edge of $3 \mathrm{eV}$

\subsubsection{Bulk versus surface Prussian blue}

Typical XANES spectra acquired in the bulk and at the surface of three Prussian blue particle aggregates are shown in Fig. 3. To increase signal-to-noise ratio and get a more statistical overview, all $\mu$ XANES spectra acquired at the surface and in bulk regions were averaged, respectively, and are shown coloured in the figure.

Spectra acquired at the surface of the aggregates are distinct from the bulk. A shift of the absorption edge towards lower energies (up to $\approx 2 \mathrm{eV}$ ) is observed, accompanied by a rounding of the post-edge and a shift of the first oscillation (stars). The overall spectral changes are similar to those observed by X-ray beam damage (Fig. 2).

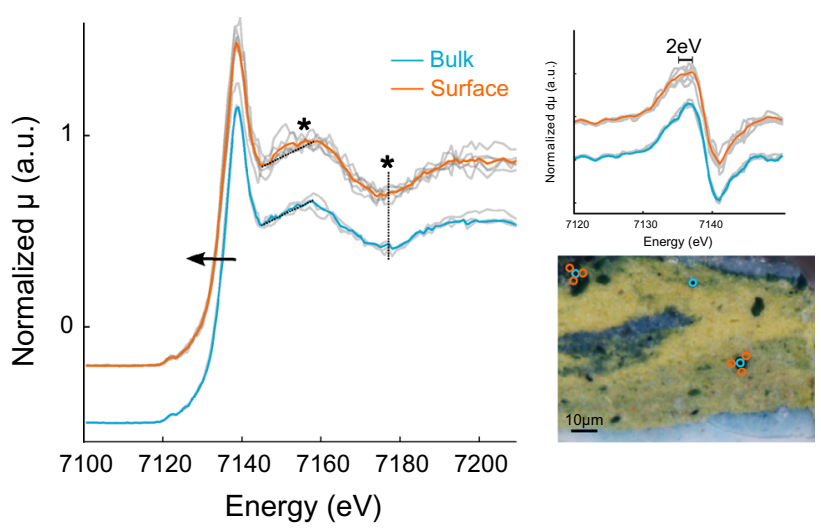

Fig. $3 \mu \mathrm{XANES}$ spectra acquired at the surface and in the bulk of Prussian blue particle aggregates. Inset: derivatives of the XANES spectra. Spectral variations between bulk and surface are denoted by arrow, stars and dotted lines 


\subsubsection{Prussian blue in diluted zones mixed with zinc white}

Spectra acquired in Prussian blue diluted areas close to, or within the zinc white layer are shown in Fig. 4, along with spectra recorded on the three Prussian blue paint mock-ups. In diluted areas of the paint sample, the number of counts was very low (green and red spot with $\approx 3000$ counts), but distinct spectral signatures could nevertheless be recognized. In the green spot located close to, but outside of the zinc white layer, the signature of Prussian blue is still visible. In the red spot located within the zinc layer however, the spectrum presents a loss of the first oscillation after the edge. The mock-up $\mathbf{P B}++\mathbf{Z n}$ shows a similar spectrum. Comparison with reference spectra acquired in previous XAS sessions indicates that this spectrum is similar to an iron oxyhydroxide such as ferrihydrite or goethite. It could be due to a complete degradation of Prussian blue already observed in long X-ray exposures of dyed cellulosic fibres [12]. The presence of ferrihydrite was already mentioned as a byproduct of Prussian blue synthesized by eighteenth's century recipes and was detected as traces in a historical paint layer containing discoloured Prussian blue [8].

Finally, the grey spectrum was acquired in a region with even lower concentration of Prussian blue embedded in a matrix principally composed of zinc white. Despite the significant flux $\left(\approx 10^{10}\right.$ photons/s), the number of counts
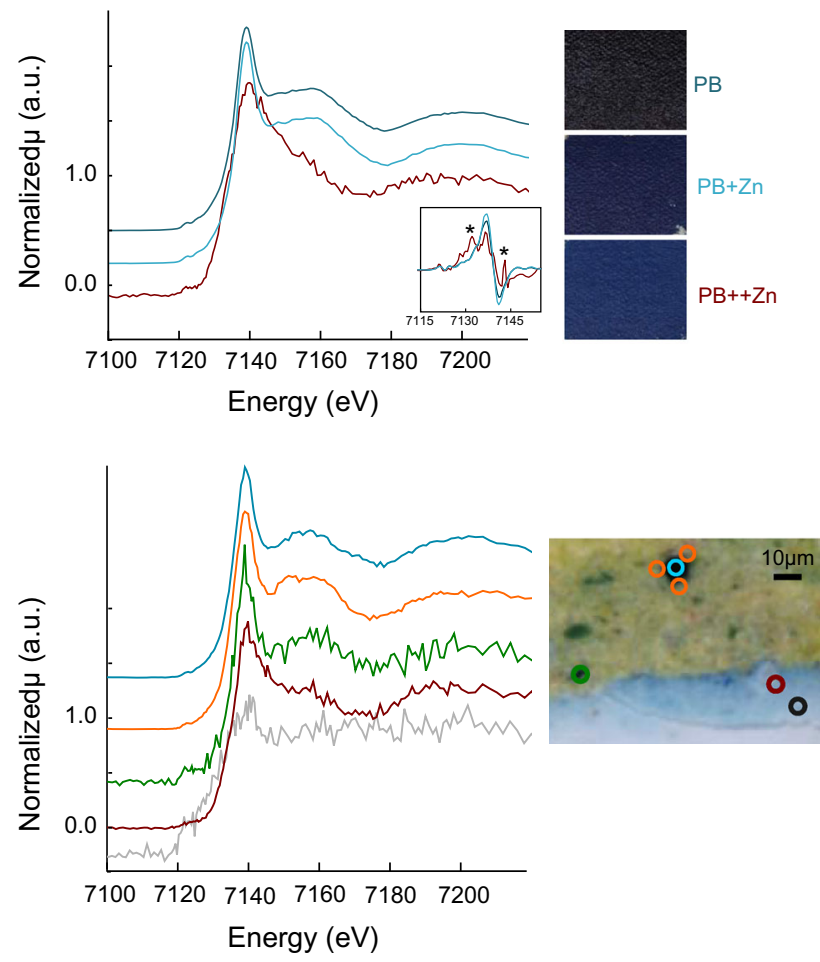

Fig. $4 \mu$ XANES spectra acquired in Prussian blue mock-ups (top) and in regions of the paint sample where Prussian blue is diluted and close to the zinc white layer (bottom) was insufficient and any attempt to increase either the flux or the exposure time would have resulted in an increase in radiation damage (the silicon drift detector was already as closest as possible to the paint). This spectrum highlights an actual challenge in the micro-analysis of paint samples containing Prussian blue or other photosensitive pigments, i.e. acquiring a sufficient statistics while keeping the photon flux and dose sufficiently low to prevent radiation damage.

\subsection{Analysis of zinc white at Zn K-edge}

Twenty-one $\mu$ XANES spectra were collected over the paint sample. To evaluate the stability of the pigment under the beam, three $\mu$ XANES spectra were collected on the same spot: two were recorded successively, followed by a third analysis performed three hours later, including a complete EXAFS spectrum at the Zn K-edge. No spectral change was observed between the three spectra (Fig. 5). The spectral signature was in good agreement with the literature and indicate that the pigment has a wurtzite-type structure with a fourfold coordination, the most common phase of $\mathrm{ZnO}$ at room temperature and pressure [13].

\subsection{Post-analysis of the paint sample and mock-ups}

\subsubsection{Raman spectroscopy of Prussian blue areas}

Potential damages in Prussian blue were further assessed by optical microscopy and Raman spectroscopy. Under dark field illumination, no discolouration of Prussian blue could be observed, whatever its local concentration or when admixed with other pigments in the sample. Raman spectroscopy of zones irradiated with X-rays was compared with untouched zones as shown in Fig. 6. No clear

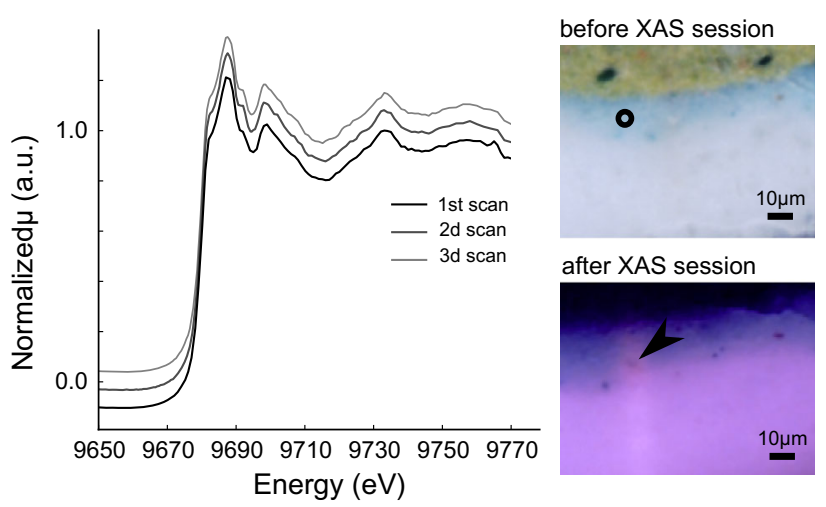

Fig. $5 \mu$ XANES spectra acquired successively on one spot (grey circle) of the zinc white layer. No spectral change is noticed; however, a visual change is observed by UV light microscopy on all spots measured by $\mu$ XANES and particularly on this spot (image with artificial colour) 


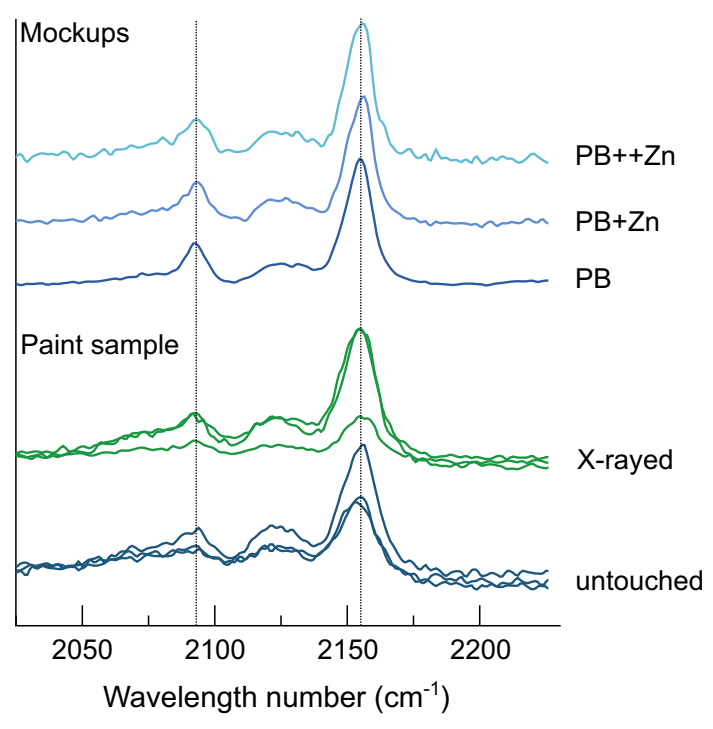

Fig. 6 Raman spectra of mock-ups and several spots measured in Prussian blue rich zones of the paint sample (X-rayed: zone that has been investigated by $\mu$ XANES at the Fe K-edge; untouched: zone not investigated by $\mu$ XANES) The spectra are zoomed on the $2000-2200 \mathrm{~cm}^{-1}$ range where the $\sigma(\mathrm{CN})$ cyano peak typical of Prussian blue is located

difference was observed, neither in the position of the $\sigma(\mathrm{CN})$ peaks nor in the ratio of their intensities. Likewise, no spectral difference was observed between the mock-ups and the paint sample.

\subsubsection{Synchrotron photoluminescence of zinc white areas}

Potential damages in the zinc white layer were further assessed by visible and UV light optical microscopy and by synchrotron photoluminescence imaging [14]. No clear damage could be detected under visible light, although some irradiated areas showed a very light yellowing. UV light microscopy revealed, however, a clear increase in photoluminescence at the $\mu$ XANES spots (Fig. 5). The excitation domain used $(450-490 \mathrm{~nm})$ corresponds to a lower energy than the zinc oxide bandgap $(370 \mathrm{~nm}$ or $3.37 \mathrm{eV}$ at room temperature). Thus, the photoluminescence observed can only be induced by the binder and not by the zinc white pigment.

Zinc oxide is a wide bandgap semiconductor whose luminescence properties are often used to evaluate defects in the crystal structure [15]. Deep-UV/visible (DUV/Vis) photoluminescence micro-imaging was implemented using an excitation at $275 \mathrm{~nm}(4.50 \mathrm{eV})$ allowing thus the excitation of $\mathrm{ZnO}$. Two high-resolution images $(289 \mathrm{~nm}$ projected pixel size) were collected in the range of the band edge emission of $\mathrm{ZnO}(370-410 \mathrm{~nm})$ and in the green range $(499-529 \mathrm{~nm})$ corresponding to defect states emission (Fig. 7). A strong decrease in photoluminescence in the band edge emission image is observed at all $\mu$ XANES spots (yellow boxes). Likewise, the photoluminescence intensity decreased at these spots in the defect state emission image, but to a less significant extent.

\section{Discussion}

\subsection{Challenge in the analysis of Prussian blue paints by $\mu$ XANES analysis}

The photosensitive pigment Prussian blue is known to be sensitive to radiation damage, especially when embedded into a substrate which itself readily reacts to light [16]. For instance, exposure of Prussian blue fibre samples to X-rays damages the pigment in two successive steps, namely the reduction of the pigment followed by its complete degradation into iron oxy-hydroxide [12]. This process has been shown to be very similar to the reduction induced by natural light ageing. In Prussian blue paint systems however, natural ageing seems to follow a different pathway because of the presence of oil that reacts differently than a cellulosic substrate. Samain et. al. proposed that Prussian blue particles undergo an oxidation in the bulk, through an exchange of hydroxyl irons or radicals with the linseed oil, together with a reduction at the surface of the particles, promoted by the photo-oxidative degradation of the fatty acids [17]. The $\mu$ XANES analyses presented here confirm a lower oxidation state at the surface of Prussian blue particle aggregates than in the bulk. However, the presence of radiation damage prevents us to confirm a chemical origin for this difference. Indeed, spectra were acquired at the surface and in the bulk in the same conditions and with the same flux (number of photons per seconds), but the amount of absorbing Prussian blue was drastically lower in surface regions. That is, the absorbed dose was significantly larger for Prussian blue at the surface than in the bulk of particle aggregates, increasing their risk of being photoreduced by the beam. Therefore, while measurements indicate a significant spectral change between PB surface and bulk areas, the fact that this difference could be an artefact due to radiation damage cannot be ruled out. This warning of mis- or over-interpretation applies here and should be kept in mind for every high-flux/ micro-spot analysis of Prussian blue in paints.

As a more positive aspect, $\mathrm{X}$-ray radiation damage is informing us qualitatively on the potential environments and/or morphology in which Prussian blue is likely to be "fragile". Comparison of $\mu$ XANES and Raman spectra between the paint sample and the unaged mock-ups revealed no spectral differences, indicating that the Prussian blue pigment is in general not degraded in the paint. These findings are in agreement with the good conservation condition of the entire painting. However, whether in the paint sample or in mock-ups, Prussian blue seems to be 


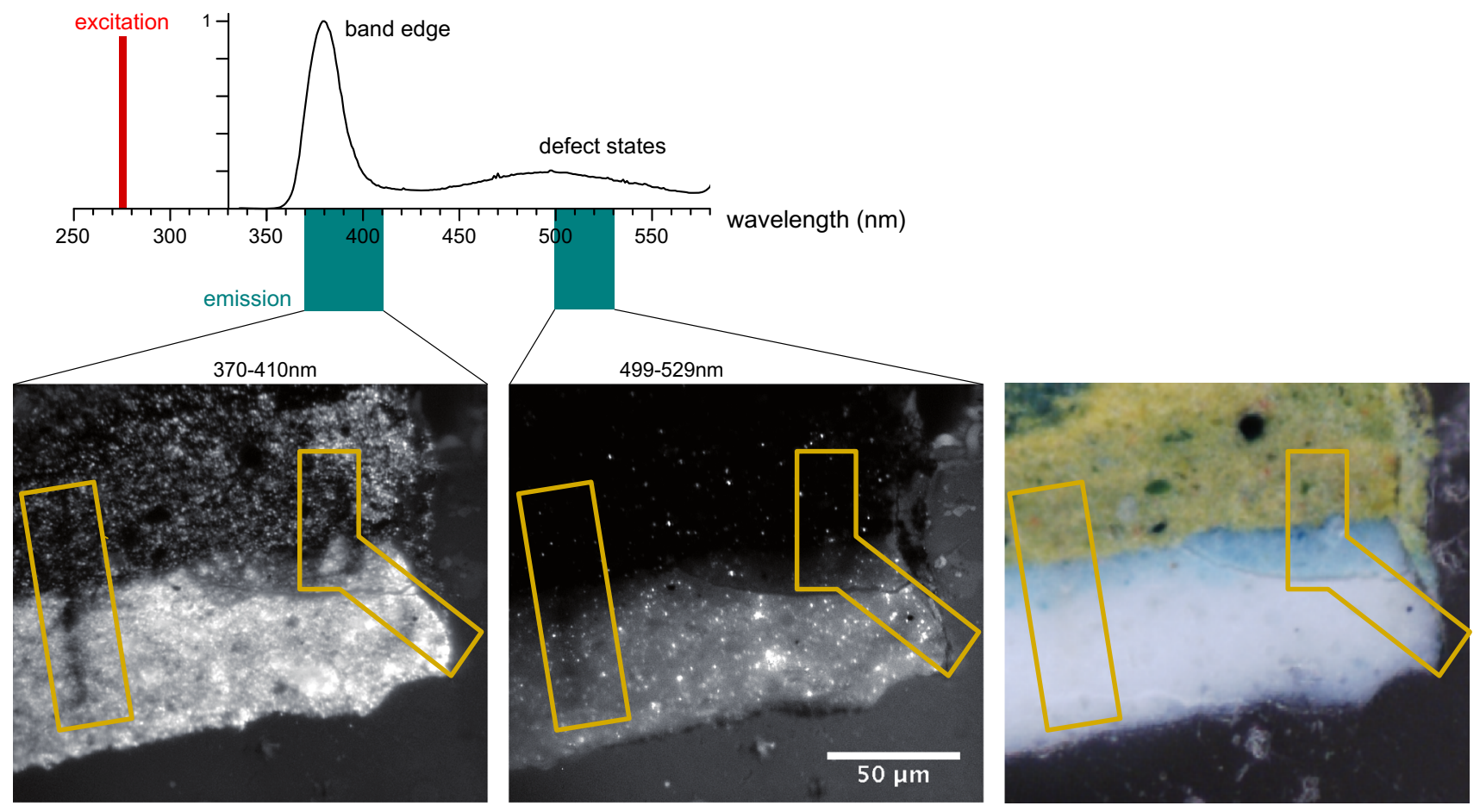

Fig. 7 Top: Photoluminescence spectrum of zinc oxide showing the band edge emission around $370 \mathrm{~nm}$ and a broad emission peak due to crystalline defects. Bottom: synchrotron photoluminescence images of the zinc white layer areas collected post- $\mu$ XANES session. Yellow areas correspond to zones analysed by $\mu$ XANES. Excitation was chosen at $275 \mathrm{~nm}$, and emission images were collected in the

fragilized in diluted regions and/or in the presence of zinc white, where it transforms into iron oxyhydroxide. By fragilized, we mean a pigment which is likely to react or even be destroyed by irradiation, whether X-rays or natural visible light. In that sense, if $\mathrm{X}$-ray radiation damage is correctly assessed and taken into account, $\mu$ XANES can be interestingly used as a predictive analytical technique.

\subsection{Challenge in detecting radiation damages and establishing a safer analytical sequence}

Detection of X-ray radiation damage was relatively easy to assess in the case of Prussian blue, since it could be estimated by comparing successive spectra acquired on the same spot (Fig. 2). But the case of zinc white shows the challenge it may be to detect radiation damages. Indeed, zinc oxide alteration under the X-ray micro-beam was not observed during $\mu$ XANES analyses at the $\mathrm{Zn}$ K-edge, rather afterwards, with a decrease in photoluminescence in the DUV range at the $\mu$ XANES spots (Fig. 7). The dual use of photoluminescence with excitation in the visible and DUV range could allow to deconvolute and detect early stages of the degradation of the oil binding medium and the zinc white pigment, respectively.
$370-410 \mathrm{~nm}$ range (left) and in the 499-529 $\mathrm{nm}$ range (middle) (intensity stretched on a logarithmic scale to enhance contrasts). Distinct changes are visible in photoluminescence (see the black lines corresponding to the successive XANES measurements) but not with visible light $(r i g h t)$

Whether for Prussian blue or zinc white, failure to detect and acknowledge $\mathrm{X}$-ray radiation damage would have highly increased the risks of misinterpreting the present analytical data or dangerously compromising forthcoming scientific campaigns on the same sample. To avoid these pitfalls, we recommend a systematic check for radiation damage during high-risk analyses, such as high photon flux or dose, along with the mention of its absence/presence in the resulting scientific publications. Ideally, the monitoring should be performed by an analysis prior, during (when possible) and after the synchrotron experiment [6]:

- Before, the analytical history of the sample should be established, as well as the chemical and morphological state of its surface (condition report) recorded. With that respect, surface techniques such as photoluminescence imaging in the UV or DUV ranges, are promising tools for organic binder or semiconducting pigments which are more and more studied using $\mu$ XANES [18].

- During, the sensitivity and reactivity of the materials should be assessed by performing several successive measurements at the same spot. This test should be performed especially in places where the risk of beam damage is high (e.g. diluted areas). 
- After, a new condition report should be performed and compared with the first one.

Besides, the systematic recording of all the campaigns of analyses realized on a sample should become a standard in the field, much as it is done for restoration campaigns of artworks with the condition report. In doubt, re-polishing of the cross section of a few micrometres in depth could help in erasing part of the sample measurement history, with the drawback that the surface topological information is irremediably lost. It is thus not always compatible with multispectroscopic imaging analytical campaigns.

Owing to the design of adequate mitigation strategies, the chronology of the analytical sequence to study a sample should be optimized by favouring measurements using lower energy range excitation (FTIR microscopy, UV photoluminescence) prior to analyses performed using higher energy (especially X-ray analyses with a micro-beam). For XANES studies, quick-EXAFS or very rapid low-resolution consecutive scans may help in gathering data before changes induced by X-ray radiation occur, although getting enough signal-tonoise ratio becomes then an issue and high-resolution spectra are usually not easily obtained with this kind of set-up.

\section{Conclusion}

$\mu$ XANES analysis of a paint sample from the beginning of the twentieth century highlighted the presence of radiation damage invisible under white light microscopy. Those changes were detected during and after experiment on Prussian blue, zinc white and the binder. For Prussian blue, it led to spectral changes similar to those found in naturally aged samples, that is a photoreduction in the pigment. This similarity highlights the danger to misinterpret analytical results but could nevertheless be used to probe Prussian blue photosensitivity, which seems to be especially high when the pigment is mixed with zinc white and diluted or with a small particle size, two cases where its interface and relationships with the surroundings becomes predominant. A novel protocol was implemented based on photoluminescence imaging with DUV and visible excitation that allowed us to detect and deconvolute post-analysis X-ray-induced radiation damages that occurred on the zinc white pigment and the oil binder.

This example highlights the fact that radiation damage may always potentially exist especially in analyses that usually require a high photon flux or for particularly photosensitive materials. It thus needs to be adequately detected and characterized. Post-measurement observations solely in the visible range is definitely not appropriate to evaluate radiation damage, which rather requires specific imaging and spectroscopic techniques targeting the possible physicochemical changes of the various painting constituents.
We wanted also to show with this example that rather than stopping any further study as soon as radiation damage is noticed, a more promising approach would consist in integrating it into the analytical pathway, by carefully checking it, measuring it and recording it for future studies on the same sample. In that respect, radiation damage should be treated similarly than other "disturbances" already accepted in the field of conservation, such as resin embedding that may disturb specific analyses (e.g. FTIR or photoluminescence studies) and cross-section preparation that may lead to mechanical displacement of material at the surface.

Acknowledgments We warmly thank Christophe Sandt for assisting Raman spectroscopy measurements, Sebastian Schoeder for fruitful comments on the work and Valerio Cugia for having prepared the mock-up paint samples. Claire Gervais acknowledges the Swiss National Science Foundation for the professorship Grant No. 138986.

\section{References}

1. M. Cotte, J. Susini, J. Dik, K. Janssens, Acc. Chem. Res. 43(6), 705 (2010)

2. L. Zanella, F. Casadio, Ka Gray, R. Warta, Q. Ma, J.F. Gaillard, J. Anal. At. Spectrom. 26(5), 1090 (2011)

3. L. Monico, K. Janssens, C. Miliani, G. Van der Snickt, B.G. Brunetti, M. Cestelli Guidi, M. Radepont, M. Cotte, Anal. chem. 85, 860 (2013)

4. L. Bertrand, M. Cotte, M. Stampanoni, M. Thoury, F. Marone, S. Schöder, Phys. Rep. 519, 51 (2012)

5. M. van Schooneveld, S. DeBeer, J. Electron Spectrosc. Relat. Phenom. 198, 31 (2015)

6. L. Bertrand, S. Schoeder, D. Anglos, M. Breese, K. Janssens, M. Moini, A. Simon. Mitigation strategies for radiation damage in the analysis of ancient materials. Trends Anal. Chem. 66, 128-145 (2015)

7. K. Patten, L. Gonzalez, C. Kennedy, D. Mills, G. Davis, T. Wess, Herit. Sci. 1, 22 (2013)

8. L. Samain, G. Silversmit, J. Sanyova, B. Vekemans, B. Gilbert, F. Grandjean, G.J. Long, P. Hermann, L. Vincze, D. Strivay, J. Anal. Atomic Spectrom. 26, 930 (2011)

9. L. Samain, B. Gilbert, F. Grandjean, G.J. Long, D. Strivay, J. Anal. Atomic Spectrom. 28, 524 (2013)

10. M. Newville, J. Synchrotron Radiat. 8, 322 (2001)

11. C. Gervais, M.A. Languille, S. Reguer, M. Gillet, E.P. Vicenzi, S. Chagnot, F. Baudelet, L. Bertrand, Appl. Phys. A 111, 15 (2013)

12. C. Gervais, M.A. Languille, G. Moretti, S. Reguer, Langmuir 31, 8168 (2015)

13. F. Decremps, F. Datchi, a. Saitta, a. Polian, S. Pascarelli, a. Di Cicco, J. Itié, F. Baudelet, Phys. Rev. B 68, 104101 (2003)

14. L. Bertrand, M. Refregiers, B. Berrie, J.P. Echard, M. Thoury, Analyst 138, 4463 (2013)

15. A.B. Djurišić, Y.H. Leung, Small 2, 944 (2006)

16. C. Gervais, M.A. Languille, S. Réguer, M. Gillet, S. Pelletier, C. Garnier, E.P. Vicenzi, L. Bertrand, J. Anal. Atomic Spectrom. 28, $1600(2013)$

17. L. Samain, F. Grandjean, G.J. Long, P. Martinetto, P. Bordet, D. Strivay, J. Phys. Chem. C 117, 9693 (2013)

18. G. der Snickt, K. Janssens, J. Dik, W. De Nolf, F. Vanmeert, J. Jaroszewicz, M. Cotte, G. Falkenberg, L. der Loeff, Anal. Chem. 84, 10221 (2012) 